\title{
Analysis and Design of an Isolated Single-Stage Three-Phase Full-Bridge with Current Injection Path PFC Rectifier for Aircraft Application
}

\author{
S. Zhao, M. Silva, J. A. Oliver, P. Alou, O. García, J. A. Cobos
}

\section{INTRODUCTION}

During the last decade, the More Electric Aircraft (MEA) concept has been gaining more and more focus both in the civil and military aircraft world [1]. This results from the replacement of pneumatic, mechanic, and hydraulic actuators by electric actuators. Thus the maintenance effort of the system is reduced and also energy conversion becomes more efficient.

All these innovations have increased the demand of electrical power in airplanes, which has prompted the incorporation of a new level of distribution power in military aircrafts. The classical aircraft distribution is a three-phase grid with 115 $\mathrm{V}$ ac at $400 \mathrm{~Hz}$ and output at $28 \mathrm{~V}$ dc. The new high DC voltage distribution is $270 \mathrm{~V}$ dc. The main advantage is that by decreasing the current level to 10 times lower than the traditional low voltage, the weight of the cabling is reduced [2], [3].

Generally speaking, an isolated three-phase AC/DC rectifier system can be achieved by : a) an active PFC rectifier stage + an isolated DC/DC stage [4]; b) a diode bridge with active power filter AC/DC stage + an isolated DC/DC stage; c) an isolated single-stage AC/DC rectifier (see Fig. 1). In (a), the complete power has to be processed through two stages, leading to high losses and thus low efficiency. However in (b), The active power filter only needs to process a small amount of total power. In this way, the global efficiency can be increased but still depends on high number of semiconductors, which is prone to lower reliability. In order to obtain high efficiency, high power density and high reliability (i.e. low number of (a)

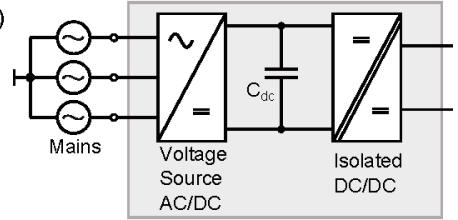

(b)

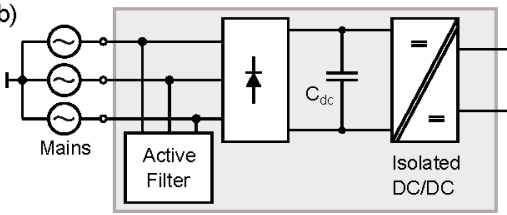

(c)

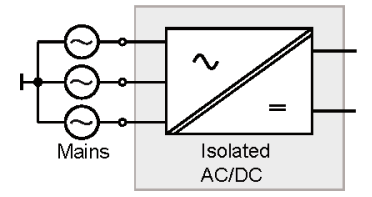

Figure 1: (a) two-stage rectifier system. (b) diode-bridge rectifier with active filter + isolated DC/DC stage. (c) single-stage isolated $\mathrm{AC} / \mathrm{DC}$ converter.

controlled semiconductors), the emergence of isolated singlestage topology is advantageous [5].

In [6] an isolated matrix-type rectifier inheriting the operating principle of full-bridge is proposed, with the merit of Zero Voltage Switching (ZVS) and single-stage isolation. It has large number of semiconductors and modulation complexity. In [7], an isolated single-stage topology is presented based on a two-switch three-phase rectifier. This topology is easy to control and it operates in discontinuous conduction mode (DCM). Thus it shows higher RMS current which can be useful for high frequency low-medium power application. An isolated single-stage Swiss-Forward topology is proposed in [5], showing lower number of high frequency transistors but higher voltage stress on semiconductors because of the forward structure.

Later proposed in [8], the rectifier stage of the $\mathrm{I}^{2} \mathrm{AFM}$ PFC rectifier consists of a passive three-phase diode bridge rectifier with an additional injection circuit: the integrated active filter (IAF), comprising a high-frequency bridge-leg with the switches $S_{1}$ and $S_{2}$, the inductance $L$ and the lowfrequency bidirectional switches $S_{a}, S_{b}$ and $S_{c}$. In order to 


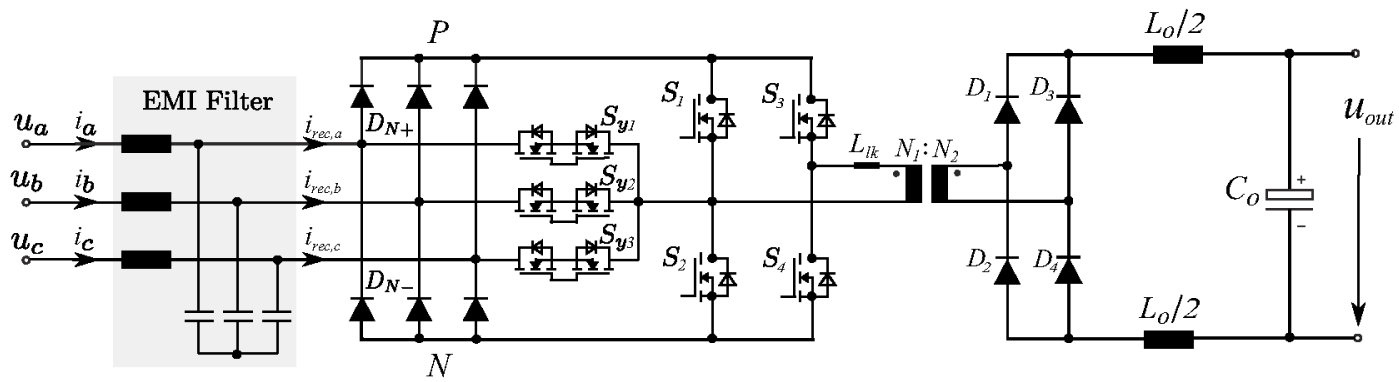

Figure 2: Proposed topology of an isolated single-stage three-phase full-bridge with current injection path PFC rectifier (IS ${ }^{2}$ FBCIP PFC Rectifier).

achieve sinusoidal input currents shape from the mains, the two switches on the half-bridge leg of the injection circuit can be controlled in such a way that a third harmonic current is forced into the phase with the smallest input absolute voltage. Besides, since the intermediate bus voltage will not be constant but vary with a sixfold mains-frequency, the phase shift in the full-bridge operation has to be adjusted to obtain a constant dc output voltage, meanwhile providing isolation. Also, a capacitor is placed in between two stages to decouple the functionality.

Based on the $\mathrm{I}^{2} \mathrm{AFM}$ PFC rectifier, an isolated single-stage three-phase full-bridge $\mathrm{PFC}$ rectifier with current injection path is proposed in this paper (denominated as IS $^{2} \mathrm{FBCIP}$ PFC Rectifier), shown in Fig. 2. This topology is an isolated single-stage rectifier, and it has ZVS feature since the fullbridge operation (by MOSFETs $S_{1}, S_{2}, S_{3}, S_{4}$ ) is integrated. Each component on the current injection path $\left(S_{y 1}, S_{y 2}\right.$ and $S_{y 3}$ ) needs to be a bidirectional switching pair since it has to present a four-quadrant behavior. On the secondary of the transformer, a diode bridge rectifier and $L-C$ output filter are used to reach a constant output voltage $u_{\text {out }}$. Compared to $\mathrm{I}^{2} \mathrm{AFM}$ PFC rectifier, less semiconductors are used, ZVS can be reached for all the semiconductors, and also the decoupling capacitor and current injection inductor are removed in the proposed topology, leading to higher efficiency and more compact design.

In Section II, the operating principle and modulation method of IS $^{2}$ FBCIP PFC rectifier are discussed including the description of ZVS realization. In Section III, a design guideline is presented for a demonstrator prototype with output power rating of $3.3 \mathrm{~kW}$, simulation results are provided to validate the functionality of the converter topology. The prototype hardware design parameters are finally described in Section IV.

\section{Operating Principle and Modulation Method}

The operating principle of the proposed rectifier shows similarity with $\mathrm{I}^{2} \mathrm{AFM}$ PFC Rectifier, being different that the operation of the current injection path is integrated into the operation of the phase-shifted full-bridge.

\section{A. Operating Principle}

To demonstrate the operating principle of the proposed rectifier, 12 sectors of the mains phase voltage are divided and shown in Fig. 3. Here sector 1 (where $u_{a}>0>u_{b}>u_{c}$ ) is used for example. As shown in Fig. 4, $u_{\text {prim }}$ depicts the voltage

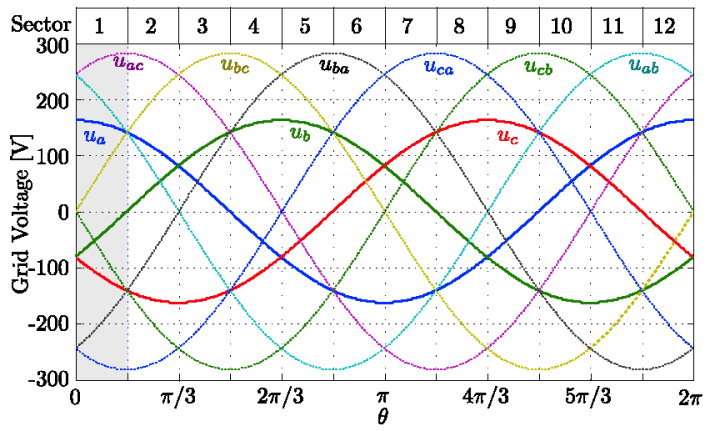

Figure 3: Sectors $1-12$ of the mains phase voltage.

applied on the primary of the transformer in each switching cycle. There are five operation states: three active states (marked as time interval $t_{1}, t_{2}$ and $t_{3}$ ) and two freewheeling states (each with time interval of $t_{4} / 2$ ). States 1,3 and 5 are active states where each phase current is demanded proportionally to its corresponding phase voltage over one switching period, shown as $i_{r e c, a}, i_{r e c, b}$ and $i_{r e c, c}$ in Fig. 4. Also the driven signals for the switches $S_{1}, S_{2}, S_{3}, S_{4}$ and the current injection switch $S_{y 2}$ are shown in Fig. 4. Driven signals for $S_{y 1}$ and $S_{y 3}$ are not shown in Fig. 4 because they are totally off in sector 1. Also, for explicit illustration, the current path for above mentioned five operation states is depicted in Fig. 5 a)-e) respectively.

By the functionality of the three-phase diode bridge $D_{N+/-}$, point $\mathrm{P}$ always presents the most positive voltage among the three phases, and likewise the most negative voltage at point $\mathrm{N}$. Specifically in the presumed sector $1, D_{a+}$ and $D_{c-}$ are always forward biased, which means that $u_{a}$ is applied at point $\mathrm{P}$ and $u_{c}$ at point $\mathrm{N}$. During time interval $t_{1}, S_{3}$ and current injection switch $S_{y 2}$ are on, thus the voltage $u_{a}-u_{b}$ is applied on the primary of the transformer. $i_{r e c, a}$ shows a discrete positive current with dc level of $I_{d c} \frac{N_{2}}{N_{1}}$ ( $I_{d c}$ is the output dc inductor current which is assumed constant in one switching period). Meanwhile the current circulates back through the injection path to phase B as $i_{r e c, b}$. Next for the freewheeling time $t_{4} / 2$, current injection switch $S_{y 2}$ is off and $S_{1}$ is on, resulting to a zero voltage on the primary of the transformer. Thus no currents are demanded from the mains and meanwhile output inductor current is freewheeling through the diode bridge on the secondary. In time interval $t_{2}, S_{4}$ is on after $S_{3}$ is turned off, thus a negative voltage is applied on the primary of the transformer with the value of $-\left(u_{a}-u_{c}\right)$. Consequently, $i_{r e c, a}$ 


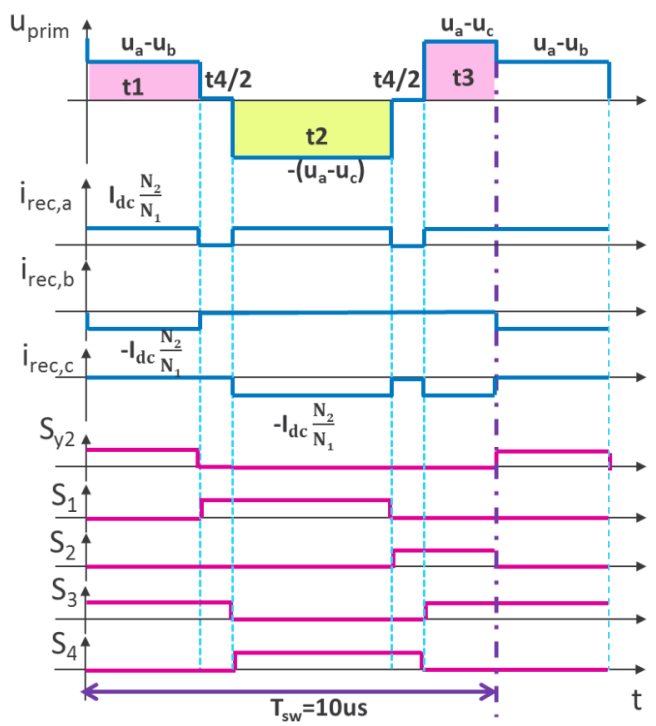

Figure 4: Operating principle of the proposed IS ${ }^{2} \mathrm{FBCIP}$ Rectifier.

presents again a discrete positive current of $I_{d c} \frac{N_{2}}{N_{1}}$ while $i_{r e c, c}$ shows a negative $-I_{d c} \frac{N_{2}}{N_{1}}$ correspondingly. At the following freewheeling time $t_{4} / 2, S_{1}$ is turned off and then $S_{2}$ is turned on. Same like before, zero voltage applied on the primary of the transformer thus there is no direct power transfer between primary and secondary. Time interval $t_{3}$ is the last state in a switching period, where a reverse voltage of the one in $t_{2}$ is applied on the transformer, aiming at balancing the flux in the transformer over one switching period. Namely in the waveform of $u_{\text {prim }}$, the integral area over time intervals $t_{1}$ and $t_{3}$ has to be equal to the area of $t_{2}$. After time interval $t_{3}$, starts again the current injection state $t_{1}$ for the next period.

Since this topology is also a buck-type rectifier topology, the definition of modulation index $M$ in [9] is still valid here. Thus, there is:

$$
M=\frac{2}{3} \cdot \frac{N_{1}}{N_{2}} \cdot \frac{u_{o u t}}{\hat{U}_{N}}
$$

where $u_{\text {out }}$ represents the dc output voltage and $\hat{U}_{N}$ represents the amplitude of the input phase voltages.

\section{B. Modulation Method}

The modulation method is the essence of the performance for the proposed rectifier topology. Two principle laws have to be satisfied while designing the modulation method:

- Transformer volt-second balance,

- Ohmic behavior for each phase (under the condition of no reactive power handling).

To ensure the transformer volt-second balance, the magnetizing flux increment and decrement have to be equalized over one switching period. This implies that (e.g. in sector 1),

$$
\left(u_{a}-u_{b}\right) \cdot t_{1}+\left(u_{a}-u_{c}\right) \cdot t_{3}=\left(u_{a}-u_{c}\right) \cdot t_{2}
$$

which derives

$$
t_{2}-t_{3}=\frac{u_{a}-u_{b}}{u_{a}-u_{c}} \cdot t_{1}
$$

Besides, for the most negative phase $\mathrm{C}$ in this sector 1 , according to the desired phase ohmic behavior, there is:

$$
t_{2}+t_{3}=\frac{M}{\hat{U}_{N}} \cdot\left|u_{c}\right| \cdot T_{s w} .
$$

Meanwhile to maintain ohmic behavior for the intermediate phase by current injection (i.e. phase B in sector 1),

$$
t_{1}=\frac{M}{\hat{U}_{N}} \cdot\left|u_{b}\right| \cdot T_{s w}
$$

can be obtained. Combining (3) and (4), there is:

$$
t_{2}=\frac{1}{2}\left(\frac{M}{\hat{U}_{N}} \cdot\left|u_{c}\right|+\frac{u_{a}-u_{b}}{u_{a}-u_{c}} \cdot \frac{M}{\hat{U}_{N}} \cdot\left|u_{b}\right|\right) \cdot T_{s w}
$$

and

$$
t_{3}=\frac{1}{2}\left(\frac{M}{\hat{U}_{N}} \cdot\left|u_{c}\right|-\frac{u_{a}-u_{b}}{u_{a}-u_{c}} \cdot \frac{M}{\hat{U}_{N}} \cdot\left|u_{b}\right|\right) \cdot T_{s w} .
$$

In the end, the total freewheeling time can be calculated as

$$
t_{4}=T_{s w}-t_{1}-t_{2}-t_{3}
$$

and each $t_{4} / 2$ will be placed between two active states.

Thus, according to Fig. 4, duty cycle for all the switches in sector 1 can be obtained:

$$
\begin{gathered}
d_{S_{y 2}}=t_{1} / T_{s w} \\
d_{S_{1}}=\left(t_{2}+\frac{1}{2} t_{4}\right) / T_{s w} \\
d_{S_{2}}=\left(t_{3}+\frac{1}{2} t_{4}\right) / T_{s w} \\
d_{S_{3}}=\left(t_{1}+t_{3}+\frac{1}{2} t_{4}\right) / T_{s w} \\
d_{S_{4}}=\left(t_{2}+\frac{1}{2} t_{4}\right) / T_{s w}
\end{gathered}
$$

\section{Zero Voltage Switching}

By the merit of the full-bridge architecture, the presence of leakage inductance in the transformer enables soft-switching for each switching transition, this almost eliminates all switching losses above certain load level [8]. In [8], ZVS is only realized in the DC/DC full-bridge stage, but not in the injection circuit. However in this proposed topology, the functionality of current injection is integrated into the full-bridge operation inside every switching period, thus all the bidirectional switching pairs $\left(S_{y 1}, S_{y 2}\right.$ and $\left.S_{y 3}\right)$ on current injection path can also realize ZVS.

As discussed in [10], for leading leg switches $S_{1} S_{2}$ and $S_{y}$, at the moment before its turn-on, it is always in an active state where the energy used to charge or discharge its output capacitance is the energy stored in the $L_{l k}$ plus the energy stored in the output filter inductor $L_{o}$. Since the energy in $L_{o}$ is quite large compared to the energy needed to charge the 


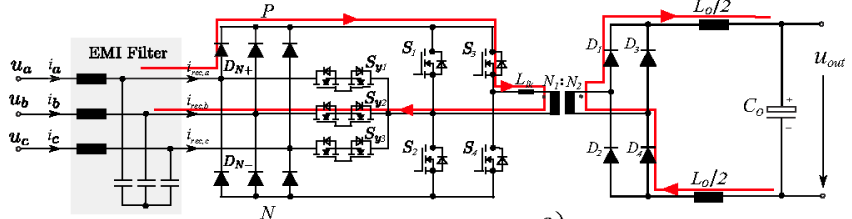

a)

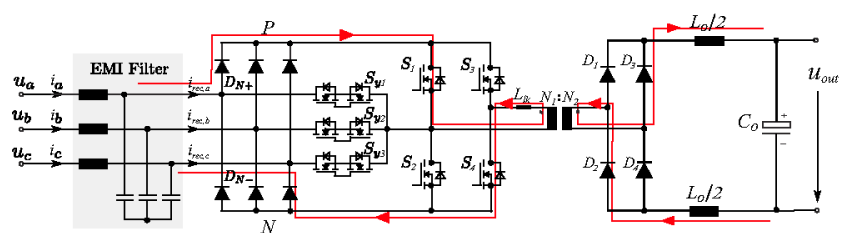

c)

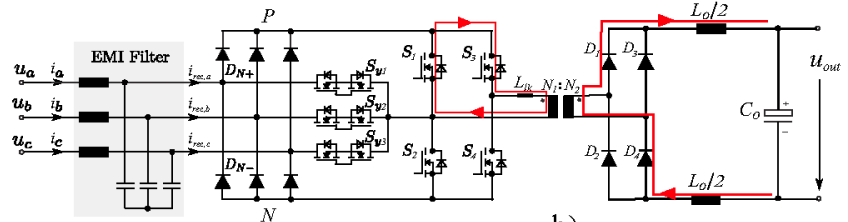

b)

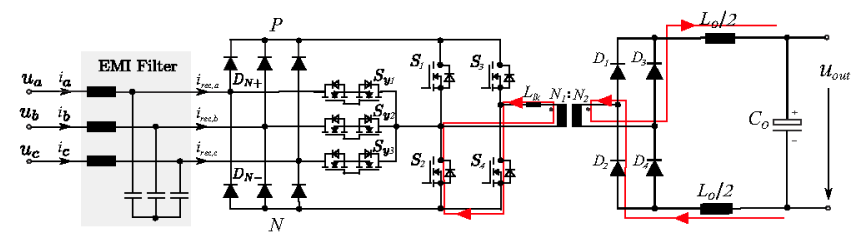

d)

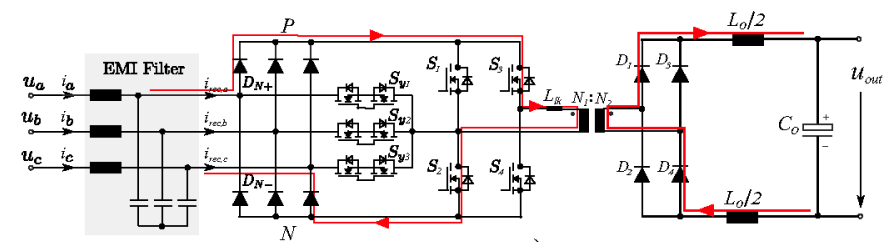

e)

Figure 5: Current path at five different operation states inside one switching cycle in sector 1.

output capacitance, it can be considered to charge as a constant current on MOSFET $C_{o s s}$. And the charging current $I_{p, \max }$ corresponds to the peak value of output inductor current ripple reflected to the primary, i.e:

$$
I_{p, \max }=I_{L, \max } \cdot \frac{N_{2}}{N_{1}}
$$

and the most strict moment to achieve ZVS regarding $u_{D S}$ is the moment when the maximum line-to-line voltage reaches its peak:

$$
U_{D S, \max }=\sqrt{3} \hat{U}_{N}
$$

For a more precise analysis, the non-linear behavior of MOSFET $C_{\text {oss }}$ should be considered. Thus the minimum deadtime applied on leading leg switches $\left(\delta_{1}\right)$ can be obtained by

$$
2 \int_{0}^{U_{D S, \max }} C_{o s s}\left(u_{D S}\right) \cdot d\left(u_{D S}\right)=I_{p, \max } \cdot \delta_{1} .
$$

The left part can be easily achieved by integrating the area below the provided $C_{o s s}-u_{D S}$ curve in MOSFET device datasheet.

on the other hand, the turn-on of the lagging leg switches $S_{3} S_{4}$ happens after a freewheeling state, which means that only the energy stored in the $L_{l k}$ is available for charging or discharging its $C_{o s s}$. Then the energy stored in $L_{l k}$ has to comply

$$
E=\frac{1}{2} L_{l k} I_{p 2}^{2}=\int_{0}^{U_{D S, \max }} C_{o s s}\left(u_{D S}\right) \cdot d\left(u_{D S}^{2}\right)
$$

where $I_{p 2}$ is the primary current at turn-on moment of the lagging leg switch which has

$$
I_{p 2}=\left(I_{L, \max }-\frac{u_{\text {out }}}{L_{o}} \frac{t_{4}}{2}\right) \cdot \frac{N_{2}}{N_{1}} .
$$

Thus, the resonance between $L_{l k}$ and $C_{\text {oss }}$ provides a sinusoidal voltage rise across the switch to be turned on. Thus the deadtime for the lagging leg switches $\left(\delta_{2}\right)$ should be at one fourth of the resonant period

$$
\delta_{2}=\frac{T_{r e s}}{4}=\frac{\pi}{2} \sqrt{L_{l k} C_{o s s}} .
$$

The simulation result showing the ZVS behavior of one switching period in sector 1 is depicted in Fig. 6. Proper deadtime has been applied to the driven signals of $S_{1}, S_{2}, S_{3}$, $S_{4}, S_{y 2+}$ (the left one of bidirectional switches pair $S_{y 2}$ ) and $S_{y 2-}$ (the right one of $S_{y 2}$ ). The shaded area implies all the five switching transitions, one can see that each switch starts to conduct current through the channel while its drain-source voltage reaches zero. A detailed zoom-in shows the MOSFET non-linear $C_{o s s}$ charging/discharging characteristic.

\section{Converter Design}

The application of the proposed rectifier topology is rooted in avionic utilities, thus in order to build a demonstrator prototype, the design specification and requirements are shown in Table I.

\section{A. Stresses on the Switches}

The transistors in this topology can be classified into two groups: those switching at high frequency (i.e. switching frequency), and those switching at an equivalently intermediate frequency between mains frequency and switching frequency. The full-bridge switches $\left(S_{1}, S_{2}, S_{3}, S_{4}\right)$ are always switching at switching frequency, and the bidirectional switching pairs on current injection path $\left(S_{y 1}, S_{y 2}\right.$ and $\left.S_{y 3}\right)$ are only switching during the sectors where its corresponding phase voltage has the minimum absolute value. The voltage stress on the fullbridge switches has an envelope of maximal line to line voltage, which gives peak voltage stress: 


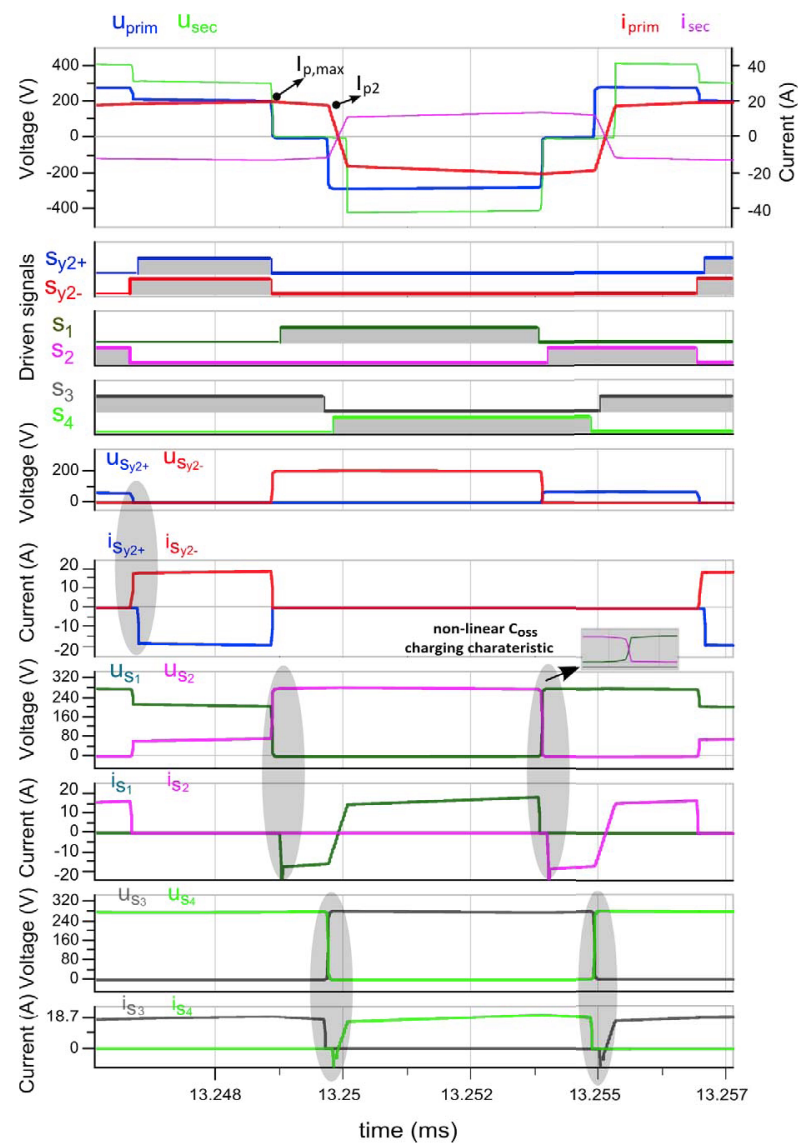

Figure 6: ZVS realization of one switching period in sector 1 .

Table I: Design specifications and requirements of the proposed isolated single-stage three-phase PFC Rectifier with current injection path

\begin{tabular}{|c|c|}
\hline Parameter & Value \\
\hline Galvanic Isolation & Yes \\
Input three-phase voltage & $115 \mathrm{~V}_{r m s, l-n}$ \\
Mains frequency & $400 \mathrm{~Hz}$ \\
Power Factor & $\geq 95 \%$ \\
THD & $\leq 5 \%$ \\
Switching frequency & $100 \mathrm{kHz}$ \\
Output voltage & $270 \mathrm{~V}$ \\
Output power rated & $3.3 \mathrm{~kW}$ \\
Semiconductor voltage derating & $75 \%$ \\
Electrolytic capacitors & Not allowed \\
Temperature derating & $70 \%$ \\
\hline
\end{tabular}

$$
\hat{u}_{S 1,2,3,4}=\sqrt{3} \cdot \hat{U}_{N} .
$$

The highest voltage stress on the switches in current injection path occurs at the moment when one phase voltage reaches its maximum amplitude and the other two are equal to half of the maximum amplitude, resulting in:

$$
\hat{u}_{S y 1,2,3}=\frac{3}{2} \cdot \hat{U}_{N} .
$$

Regarding the current stress, the behavior of the two legs (leg $S_{1}$ and $S_{2}$, leg $S_{3}$ and $S_{4}$ ) are not the same because: $S_{3}$ and $S_{4}$ are always conducting current alternatively inside each switching cycle; while $S_{1}$ and $S_{2}$ are not, since $S_{1}, S_{2}$ together with current injection path $S_{y}$ are conducting current alternatively inside every switching cycle.

The average and RMS currents through the switches on current injection path over one mains period are derived respectively:

$$
I_{S_{y 1,2,3}, R M S}=\sqrt{\frac{(2-\sqrt{3}) M}{2 \pi}} \cdot \frac{N_{2}}{N_{1}} I_{d c} .
$$

Thus, average and RMS currents through the switches $S_{1}$ and $S_{2}$ are derived:

$$
I_{S_{1,2}, R M S}=\sqrt{\frac{3}{\pi}(0.5236-0.134 M)} \cdot \frac{N_{2}}{N_{1}} I_{d c} .
$$

Since $S_{3}$ and $S_{4}$ are alternatively sharing the current $\frac{N_{2}}{N_{1}} I_{d c}$ inside every switching cycle, also its on-time is symmetrical during every $30^{\circ}$-sector, which yields to the RMS currents of $S_{3}$ and $S_{4}$ :

$$
I_{S_{3,4}, R M S}=\frac{\sqrt{2}}{2} \cdot \frac{N_{2}}{N_{1}} I_{d c} .
$$

\section{B. Input and Output Diode Rectifier}

The three-phase diode bridge $D_{N+}$ and $D_{N-}$ are low frequency devices which switch at mains frequency. The voltage stress on the three-phase diode rectifier is equal to the maximal line-to-line input voltage, giving the highest voltage stress:

$$
\hat{u}_{D_{N \pm}}=\sqrt{3} \cdot \hat{U}_{N} .
$$

Thus, Si-diodes with a low forward voltage drop and a blocking voltage of at least $400 \mathrm{~V}$ should be selected.

The output diode rectifier is working at switching frequency, and its voltage stress corresponds to the voltage stress in the low frequency input diodes multiplied by the turns-ratio of the transformer, which is,

$$
\hat{u}_{D 1,2,3,4}=\frac{N_{2}}{N_{1}} \cdot \sqrt{3} \cdot \hat{U}_{N} .
$$

Regarding the current stress, the average and RMS currents through the three-phase diode bridge are respectively given by:

$$
\begin{gathered}
I_{D_{N \pm}, a v g}=\frac{\sqrt{3} M}{2 \pi} \cdot \frac{N_{2}}{N_{1}} I_{d c} \\
I_{D_{N \pm}, R M S}=\sqrt{\frac{\sqrt{3} M}{2 \pi}} \cdot \frac{N_{2}}{N_{1}} I_{d c} .
\end{gathered}
$$

The current through the output diode rectifier is related with the current through full-bridge switches $S_{3}$ and $S_{4}$, thus its average and RMS currents are respectively given by:

$$
\begin{gathered}
I_{D_{1,2,3,4}, a v g}=\frac{1}{2} \cdot I_{d c} \\
I_{D_{1,2,3,4}, R M S}=\frac{\sqrt{2}}{2} \cdot I_{d c} .
\end{gathered}
$$




\section{Transformer}

The transformer is one of the most important components in the proposed topology, since the modulation of the rectifier is tightly connected to the presence of the transformer. This is different from the proposed $\mathrm{I}^{2} \mathrm{AFM}$ PFC Rectifier in [8], where the functionality of the full-bridge stage is decoupled from the rectifier stage with a capacitor in between. Besides, based on the desired ZVS feature, the leakage inductance $L_{l k}$ of the transformer has to be decided in order to achieve a certain ZVS range.

The shape of the transformer primary voltage inside one switching cycle of sector 1 is illustrated as $u_{\text {prim }}$ in Fig. 4. Even though in state 1 voltage stress on transformer is smaller compared to states 3 and 5, the maxim flux density $\hat{B}$ is defined when the primary voltage reaches peak value of the maximum line-to-line voltage, i.e. $\theta=\frac{n \pi}{6}(\mathrm{n}=1,3,5 \ldots)$. Also at these moments, time interval for state $1\left(t_{1}\right.$ in Fig. 4) equals to zero since the average current demanded by the phase with minimum absolute value will be 0 . Therefore, time interval for state 3 ( $t_{2}$ in Fig. 4 ) and state $5\left(t_{3}\right.$ in Fig. 4 ) will be equal. Thus the rectifier will work like a classical phase-shifted fullbridge [10] which gives the maximum flux density:

$$
\hat{B}=\frac{\sqrt{3} \hat{U}_{N} \cdot T_{s w} / 2}{N_{1} \cdot A_{\text {core }}}
$$

where $A_{\text {core }}$ represents the cross section area of the chosen core.

Also based on (1), the turns ratio of the transformer can be represented:

$$
N_{1} / N_{2}=\frac{3}{2} \cdot M_{\max } \cdot \frac{\hat{U}_{N}}{u_{\text {out }}} .
$$

Usually maximum modulation index $M_{\max }$ for nominal output power is set around 0.9 to leave enough margin for the control [11] and also take into consideration of the duty cycle loss from applied duty cycle on the primary to the effective duty cycle on the secondary for full-bridge topology [10].

To achieve ZVS, Sufficient leakage inductance $L_{l k}$ as discussed in Section II-C can be achieved by adding insulator between primary winding and secondary winding. Finally the transformer winding construction is described in Table II, which after measurement shows magnetizing inductance $L_{m a g}=1.2 \mathrm{mH}$ and leakage inductance $L_{l k}=2.8 \mathrm{uH}$ on primary side.

\section{Input and Output Filter}

The input filer has to be designed in a way that the EMC measurement complies with the military standard MIL-STD$461 \mathrm{E}$ [12], this imposes more difficulty in input filter design compared to CISPR standard [13] in industrial application. In CISPR standard, the EMI spectrum has limit in the range of $150 \mathrm{kHz}-30 \mathrm{MHz}$, thus the switching frequency can be chosen below $150 \mathrm{kHz}$ with the grid frequency being $50 / 60 \mathrm{~Hz} \mathrm{so}$ that the current spectral component at switching frequency (or even the first several harmonics of switching frequency) doesn't have to be considered for the input filter design [11]. However, in MIL-STD-461E, the limit starts from $10 \mathrm{kHz}$ (see Fig. 8) which is much smaller compared to the starting frequency of

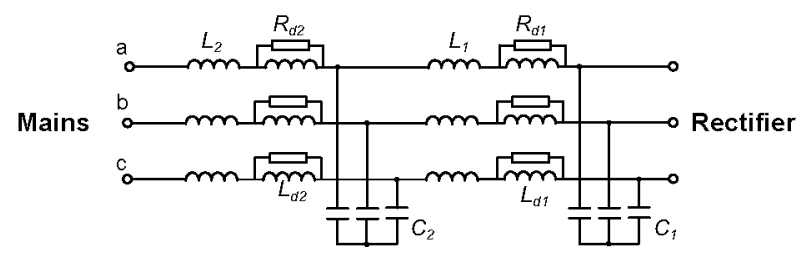

Figure 7: Circuit diagram of the two-stage input EMI filter.

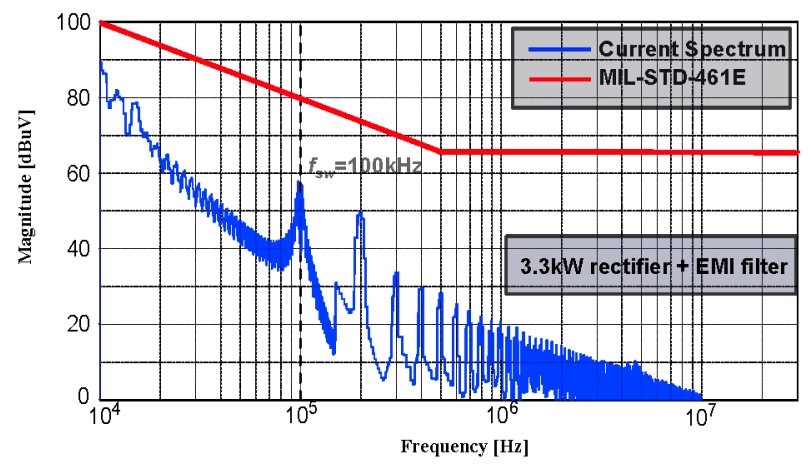

Figure 8: Input Current Spectrum of the $3.3 \mathrm{~kW}$ IS $^{2}$ FBCIP PFC Rectifier (complying with the MIL-STD-461E standard).

CISPR, meanwhile being closer to the mains frequency ( 400 $\mathrm{Hz}$ ). Therefore, the switching frequency has to be placed inside the MIL-STD-461E frequency range and moreover, the EMI filter has to be designed to attenuate the first harmonic of switching frequency.

Furthermore, buck-type rectifiers feature pulsating input currents, thus compared to boost-type rectifiers, more EMI filter effort is needed. Shown in Fig. 7, a two-stage filter with $R-L_{d}$ series damping [14] is employed, noting that the first stage filter capacitors $C_{1}$ have to placed closely to the rectifier input stage in order to maintain a low commutation inductance [11].

Output filter $L_{o}-C_{o}$ can be designed based on dc inductor current ripple and output voltage ripple values. Assuming that the output voltage $\left(u_{o u t}\right)$ ripple is negligible accross $C_{o}$, the maximum inductor current ripple happens at moments $\theta=\frac{n \pi}{6}(\mathrm{n}=1,3,5 \ldots)$ where the phase with intermediate voltage value is crossing zero thus no current demanded from current injection path, which gives the maximum inductor current ripple value:

$\Delta i_{L, p p, \max }=\frac{u_{\text {out }} \cdot\left(1-\frac{\sqrt{3}}{2} M\right)}{2 \cdot f_{s w} \cdot L_{o}}=\frac{3 M \hat{U}_{N}}{4 L_{o} f_{s w}} \frac{N_{2}}{N_{1}}\left(1-\frac{\sqrt{3}}{2} M\right)$.

\section{E. Simulation Results}

Since the proposed rectifier is a PWM buck-type rectifier, it demands a pulsating input current from the mains [5]. Consequently an EMI input filter is is deisgned to make the demanded current comply with military standard MIL-STD461E [12]. Performed in simulator GeckoCIRCUITS [15], LISN (Line Impedance Stabilization Network) measurement of the input current spectrum in comparison with MIL-STD461E is shown in Fig. 8. It can be seen that the designed EMI filter for this $3.3 \mathrm{~kW}$ rectifier can comply with MIL-STD-461E. 


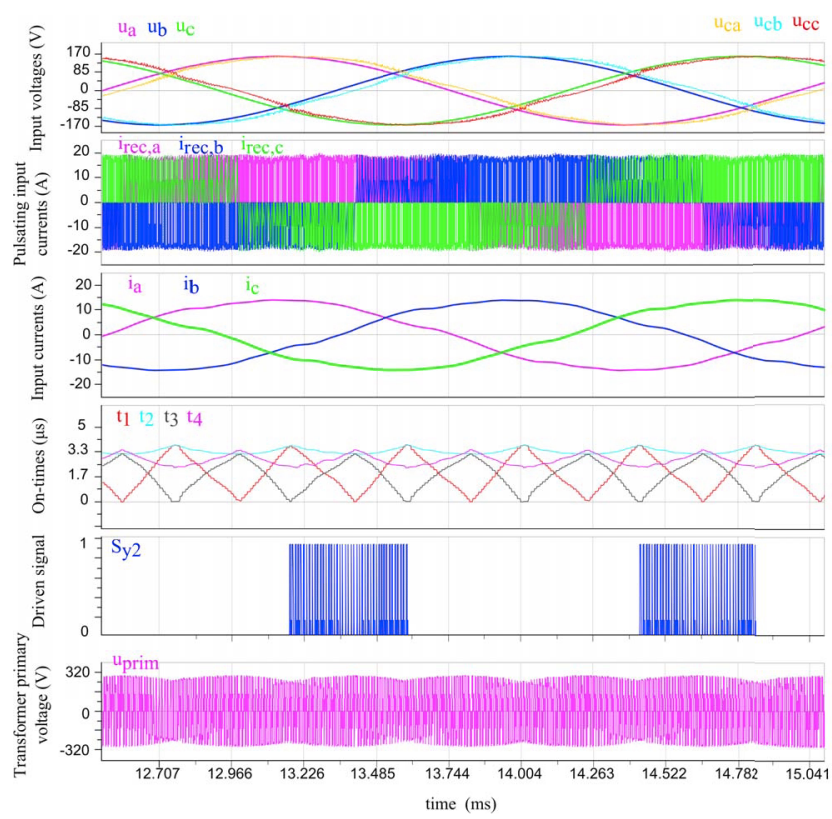

Figure 9: Simulated waveforms of the proposed IS ${ }^{2}$ FBCIP PFC Rectifier.

A simulation result of the overall performance of the rectifier with its EMI filter is shown in Fig. 9, working at $P_{o, \text { nom }}=3.3$ $\mathrm{kW}, N_{1} / N_{2}=1 / 1.5, M=0.8$ together with input and output filter value listed in Table II. One can see that, according to threephase voltages at the input capacitors of the rectifier $\left(u_{c a}\right.$, $u_{c b}$ and $\left.u_{c c}\right)$, a three-phase pulsating current $\left(i_{r e c, a}, i_{r e c, b}\right.$ and $\left.i_{r e c, c}\right)$ are demanded by the modulation scheme of the rectifier, which is in phase with capacitor voltages. Input currents from the mains are filtered by the EMI filter placed before the rectifier, thus three-phase currents $\left(i_{a}, i_{b}\right.$ and $\left.i_{c}\right)$ show good THD and PF with respect to $u_{c a}, u_{c b}$ and $u_{c c}$. Times intervals $t_{1}, t_{2}, t_{3}$ and $t_{4}$ are discussed in II-B, it is obvious that $t_{1}$ shows a third-harmonic behavior which indicates the ontime of the third-harmonic injection switching pairs. Driven signal $S_{y 2}$ on third-harmonic injection path is active only when phase B carries the minimum absolute voltage. Voltage across the transformer primary side $\left(u_{\text {prim }}\right)$ shows switching characteristic and also with an enveloped of maximum line-toline voltage defined by the three-phase diode bridge.

\section{Prototype Design}

In order to validate the design of the rectifier system, a $3.3 \mathrm{~kW}$ rectifier including EMI filter has been designed. Key components like semiconductors have been chosen, also the transformer and inductors have been designed, wound and measured. Design of the mechanical layout is in the process. The control stage is implemented by a TMS320F28377D dual-core microcontroller. A list of the main components is provided in Table. II, with the corresponding losses estimation listed in Fig. 10. It shows an overall efficiency of $95.1 \%$, which is moderate compared to the state-of-the-art rectifier topologies with isolation [5], [8], [11]. This is due to the stricter military EMI standard and also the semiconductor voltage and temperature derating applied according to Table I.
Table II: List of component employed in the prototype of the proposed isolated single-stage three-phase PFC Rectifier with current injection path

\begin{tabular}{|c|c|}
\hline Component & Description \\
\hline$L_{1}=L_{2}=200 \mu \mathrm{H}$ & $\begin{array}{l}3 \text { stacked toroid powder cores } \\
58547, \mathrm{~N}=22, \text { solid wire of } \\
1 \mathrm{~mm} \text { diameter } 4 \text { in parallel } \\
2 \mathrm{X} 1.5 \mu \mathrm{F} \mathrm{B} 32923 \text { ( } 305 \mathrm{~V} \text { ac) } \\
\text { film capacitor }\end{array}$ \\
\hline$L_{d 1}=L_{d 2}=100 \mu \mathrm{H}$ & $\begin{array}{l}1 \text { toroid powder core } 58548 \\
\mathrm{~N}=29, \text { solid wire of } 0.9 \mathrm{~mm} \\
\text { diameter } 4 \text { in parallel }\end{array}$ \\
\hline$R_{d 1}=R_{d 2}=25 \Omega$ & $\begin{array}{l}\text { CRCW251224R9 (1W) thick film } \\
\text { SMD resistor }\end{array}$ \\
\hline Input diodes $D_{N+} D_{N-}$ & $\begin{array}{l}400 \mathrm{~V} / 30 \text { A STTH30R04 ultra } \\
\text { fast recovery diode }\end{array}$ \\
\hline Current injection path $S_{y 1-3}$ & $\begin{array}{l}650 \text { V CoolMOS C6 series } \\
\text { (IPW65R037C6) }\end{array}$ \\
\hline Full-bridge $S_{1-4}$ & $\begin{array}{l}650 \text { V CoolMOS C7 series } \\
\text { (IPW65R045C7) }\end{array}$ \\
\hline Output diodes $D_{1-4}$ & $\begin{array}{l}1200 \text { V/43 A C4D30120D } \\
\text { SiC Schottky diode }\end{array}$ \\
\hline Transformer & $\begin{array}{c}\text { Ferrite core PM } 87 \text { material N27, } \\
N_{1} / N_{2}=10 / 15 \text {, primary Litz wire } \\
200 \mathrm{X} 0.07 \mathrm{~mm} 10 \text { in parallel, } \\
\text { insulator material Kapton of } \\
\text { horizontal width } 6.5 \mathrm{~mm} \text {, } \\
\text { secondary Litz wire } 200 \mathrm{X} 0.07 \mathrm{~mm} \\
4 \text { in parallel }\end{array}$ \\
\hline Output inductor $L_{o}=2 \mathrm{X} 100 \mu \mathrm{H}$ & $\begin{array}{c}\text { Ferrite core ETD59 material N87, } \\
\text { airgap } 1.2 \mathrm{~mm}, 15 \text { turms, solid wire } \\
\text { of } 1.2 \mathrm{~mm} \text { diameter } 8 \text { in parallel } \\
5 \times 40 \mu \mathrm{F} \text { B32776 ( } 450 \mathrm{~V} \mathrm{dc}) \\
\text { film capacitor }\end{array}$ \\
\hline
\end{tabular}

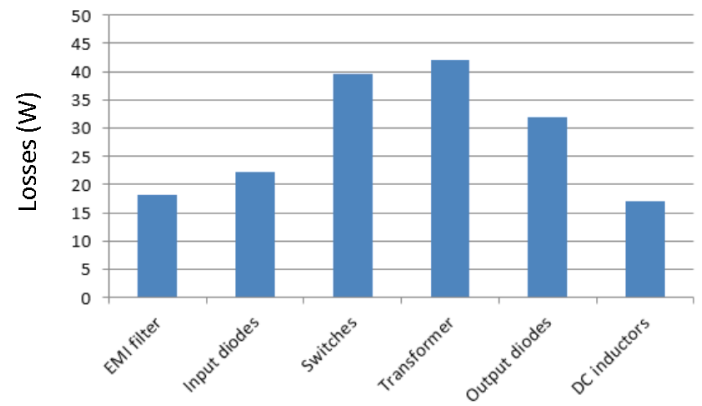

Figure 10: Distribution of losses estimation for the prototype design at $P_{o, n o m}=3.3 \mathrm{~kW}$

\section{CONCLUSION}

This paper presented a new isolated single-stage threephase full-bridge with current injection path PFC rectifier (denominated as IS ${ }^{2}$ FBCIP PFC Rectifier). Its operating principle, modulation method and ZVS feature have been discussed and the difference regarding $\mathrm{I}^{2} \mathrm{AFM}$ PFC rectifier has been addressed. Also a design guideline and simulation results are provided to validate the functionality of the proposed topology. Main components in the experimental prototype is decided. This IS ${ }^{2}$ FBCIP PFC Rectifier features an indirect matrix type converter, which is an integration of three-phase diode bridge with current injection path and a full-bridge stage without intermediate energy storage capacitor. The major advantages of the IS $^{2} \mathrm{FBCIP}$ PFC Rectifier are 
the relatively low number of switching components, low implementation effort and its ZVS feature which leads to a high overall efficiency. 